\title{
A New Image Restoration Algorithm Based on Mathematical Morphology and Wavelet Neural Network
}

\author{
Yan Shen,Ming Liu \\ College of Science \\ Harbin Engineering University \\ Harbin, 150001,China \\ shenyan@hrbeu.edu.cn
}

\author{
Jinbao Wang, Wenlu Zhou \\ College of Science \\ Harbin Engineering University \\ Harbin, 150001, China
}

\begin{abstract}
Wavelet neural network (WNN) is introduced into the field of image restoration due to the excellent local feature and adaptive ability. The procedure of restoration can be looked as an approximating procedure from noisy image to original image. The better WNN has approximation performance, the better restoration performance. With the help of wavelet neural network and mathematical morphology, a new image restoration algorithm is proposed. It can effectively maintain the image edges and details. In order to overcome the shortcomings of the filter with fixed structure, amoeba structure element is presented based on mathematical morphology. Image data is extracted by amoeba structure element, and input into the wavelet neural network.The WWN is trained by BP algorithm in batch mode training, which adjusts the wavelet coefficient and network weights adaptively.The experimental results showed that the approach proposed can preserve fine details and excellent fidelity and is better than general denoising methods.
\end{abstract}

Keywords-Sea clutter, Wavelet neural network, Amoeba structure element, Image restoration

\section{INTRODUCTION}

Image restoration is a kind of digital image processing technology to improve image quality. An image degradation model can be established according to prior knowledge of image degradation. Based on the image degradation model, the image quality can be improved by adopting various inverse degradation processing methods. Whether the best image restoration being get can be judged by some criterions. The process of image restoration can be seen as follows: finding out degeneration causes->establishing degradation model- $>$ reverse evolution->image restoration. The key problem of image restoration lies in establishing degradation model.

At present,many methods of image restoration of sea clutter are put forward to from the perspective of signal processing.But sea clutter signals have very strong correlation,such as effects of sea spike, which leads to sea clutter can not be inhibited completely from the perspective of signal processing. A sea clutter suppression technology based on image processing is given by another way in this paper. The influence of the sea clutter image caused by noises can be eliminated effectively by this method, and the target image can be separated from the sea clutter images accurately.

\section{IMAGE RESTORATION TECHNOLOGY BASED ON MATHEMATICAL MORPHOLOGY AND WAVELET NEURAL NETWORK}

\section{A. Amoeba structure element}

In this paper a new type of dynamic structure elements is designed according to the physical structure of amoebaamoeba structure element. It is a kind of filter which can automatically adjust shapes and sizes of structure elements according to the content of the image.

The shape of amoeba structure element is obtained by amoeba distance calculation, which is different from stationary structure element, and the length of path $\sigma$ is defined as follow:

$$
L(\sigma)=\sum_{i=0}^{n-1}\left[1+\lambda_{1} d_{\text {pixel }}\left(x_{i}, x_{i+1}\right)\right]
$$

In which, $d_{\text {pixel }}\left(x_{i}, x_{i+1}\right)$ is the distance between image pixel point $x_{i}$ and image pixel point $x_{i+1}$; $\sigma=\left(x=x_{0}, x_{1}, \cdots, x_{n-1}, x_{n}=y\right)$ is the path.

Between pixel point $\mathrm{x}$ and $\mathrm{y} ; n$ is the number of path $\sigma$. Because $\lambda_{1}$ is a real number, the amoebas distance with parameter $\lambda_{1}$ is defined as:

$$
\left\{\begin{array}{l}
d_{\lambda_{1}}(x, x)=0 \\
d_{\lambda_{1}}(x, y)=\min L(\sigma)
\end{array}\right.
$$

There is a strong correlation between the geometric distance expressed by amoebas distance and the gray distance of image pixel point. Following with the regulation for parameter $\lambda_{1}$, image gradient information can be expressed clearly. The amoeba structure element has been defined as follow:

$$
\operatorname{Amoeba}(x)=\left\{y \mid d_{\lambda_{1}}(x, y) \leq r\right\}
$$

In which, $r$ is a real number and is the radius of amoeba structure element, through adjusted the $r$, An amoeba-like structure elements can be divided into different scales, This is like from center pixels to start the near-far distinguish different levels, And each layer has different weights, the weights of the different scales are defined as follows: 


$$
w_{i}=\eta \frac{\frac{1}{b_{i}}}{\sum_{j=1}^{r} \frac{1}{b_{j}}} \quad i=1,2, \cdots, r
$$

In which, $w_{i}$ can be described as the weights of ith scales, $\eta$ is a positive number, which can be adjusted through the gradient change,

The self-adaptive behavior of amoeba structure kernel is shown in Figure.1.

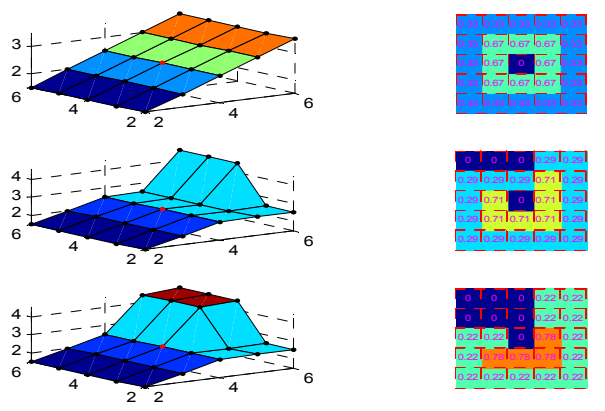

Figure 1. The self-adaptive behavior of amoeba structure kernel

In order to show the adaptive behavior of amoeba structure kernel better, partition of the scale of amoeba structure elements corresponding to different surface image can be calculated by using amoeba structure element distance. An adjusted sea wave image with $200 \times 200$ pixel and 1600 blocks (each block is in $5 \times 5$ pixel ) is given as follows, then amoeba structure element corresponding to every block center can be get based on amoeba structure kernel(that is each center of block ), in Fig.2.
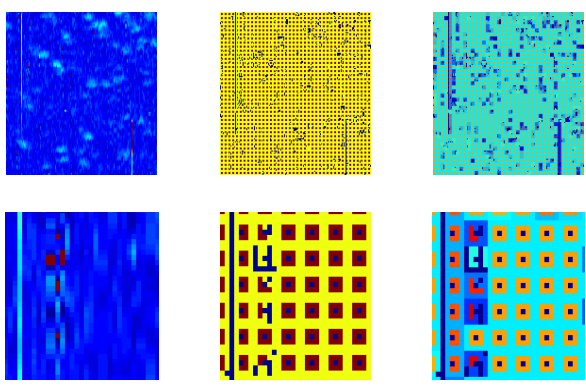

Figure 2. amoeba structure kernel

In Figure.2.in the first row there are sea wave image, extension of amoeba structure elements and their partition of the scale as well as image of amoeba structure elements after weight value set, and in the second row there are parts of amplified images respectively corresponding to the above images which are in the first row.

\section{$B$. Image restoration algorithm based on wavelet neural network}

1) Wavelet Neural Network theory
The used WNN in this paper has the following form:

$$
\begin{gathered}
\Psi_{a_{j}, b_{j}}\left(n e t_{j}^{p}(t)\right)=\Psi\left[\frac{n e t_{j}^{p}(t)-b_{j}(t)}{a_{j}(t)}\right] \\
n e t_{j}^{p}(t)=\sum_{k=1}^{m} w_{j k}(t) x_{k}^{p}(t) \\
y_{i}^{p}(t)=f\left[\sum_{j=1}^{N} w_{i j}(t) \Psi_{a_{j}, b_{j}}\left(\sum_{k=1}^{m} w_{j k}(t) x_{k}^{p}(t)\right)\right] \\
E(t)=\frac{1}{2} \sum_{p=1}^{M} \sum_{i=1}^{n}\left[d_{i}^{p}-y_{i}^{p}(t)\right]^{2}
\end{gathered}
$$

The structure of the used network is shown as follow:

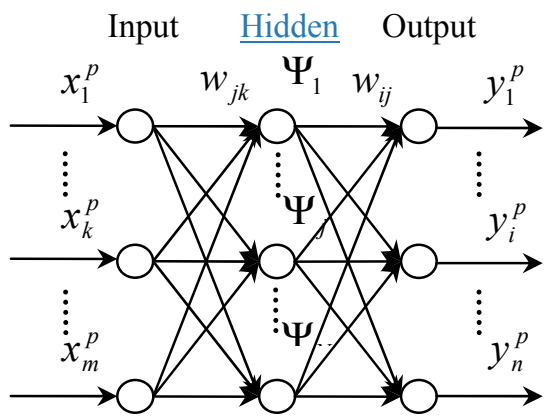

Figure 3. the model of the wavelet neural network

In this paper Marr wavelet function is Selected as wavelet function, the formula of Marr wavelet function is shown as follows:

$$
\Psi(x)=\left(1-\frac{x^{2}}{\varepsilon^{2}}\right) \exp \left(-\frac{x^{2}}{2 \varepsilon^{2}}\right)
$$

After setting the initial weights of the network and inputting training samples, the output of wavelet network can be calculated by using the current neural network parameters .It can optimize the network through the following formula:

$$
\begin{aligned}
& \frac{\partial E(t)}{\partial w_{j k}(t)}=-\sum_{p=1}^{M} \sum_{i=1}^{n}\left(d_{i}^{p}-y_{i}^{p}(t)\right) w_{i j}(t) \Psi_{a_{j}, b_{j}}^{\prime}\left(n e t_{j}^{p}(t)\right) x_{k}^{p}(t) / a_{j}(t) \\
& \frac{\partial E(t)}{\partial w_{i j}(t)}=-\sum_{p=1}^{M}\left(d_{i}^{p}-y_{i}^{p}(t)\right) \Psi_{a_{j}, b_{j}}\left(\text { net }_{j}^{p}(t)\right) \\
& \frac{\partial E(t)}{\partial a_{j}(t)}=\sum_{p=1}^{M} \sum_{i=1}^{n}\left(d_{i}^{p}-y_{i}^{p}(t)\right) w_{i j}(t) \Psi_{a_{j}, b_{j}}^{\prime}\left(\text { net }_{j}^{p}(t)\right)\left(\frac{\text { net }_{j}^{p}(t)-b_{j}(t)}{a_{j}(t)}\right) / a_{j}(t) \\
& \frac{\partial E(t)}{\partial b_{j}(t)}=\sum_{p=1}^{M} \sum_{i=1}^{n}\left(d_{i}^{p}-y_{i}^{p}(t)\right) w_{i j}(t) \Psi_{a_{j}, b_{j}}^{\prime}\left(n e t_{j}^{p}(t)\right) / a_{j}(t)
\end{aligned}
$$

The parameters of wavelet neural network is adjusted by the following formula:

$$
\begin{aligned}
& w_{j k}(t+1)=w_{j k}(t)-\eta \frac{\partial E(t)}{\partial w_{j k}(t)}+\mu \Delta w_{j k}(t) \\
& w_{i j}(t+1)=w_{i j}(t)-\eta \frac{\partial E(t)}{\partial w_{i j}(t)}+\mu \Delta w_{i j}(t)
\end{aligned}
$$




$$
\begin{aligned}
& a_{j}(t+1)=a_{j}(t)-\eta \frac{\partial E(t)}{\partial a_{j}(t)}+\mu \Delta a_{j}(t) \\
& b_{j}(t+1)=b_{j}(t)-\eta \frac{\partial E(t)}{\partial b_{j}(t)}+\mu \Delta b_{j}(t)
\end{aligned}
$$

Where $\eta$ represents the learning rate of wavelet neural network, $\mu$ represents the momentum coefficient.

\section{2) Image restoration algorithm}

Approximate estimation $\hat{\boldsymbol{f}}$ of original image can be acquired from given degraded image $\boldsymbol{g}$ according to degradation model of image restoration. Training samples by using map of wavelet neural network ,then the nonlinear mapping relationship between euclid space $R^{m}$ and euclid space $R^{n}$ is set up, which is the nonlinear mapping relationship between degraded image $\boldsymbol{g}$ and approximate estimation $\hat{\boldsymbol{f}}$ of original image.

Image restoration algorithm based on wavelet neural network as follows:

(1) Select part of degraded image ${ }^{g}$ which has relatively small noise interference from givens as training samples, and training samples are divided into flat and edge parts by amoeba operator. $\left(X^{p}, D^{p}\right)$ are vector pairs of flat part, and $\left(X^{\prime p}, D^{\prime p}\right)$ are vector pairs of edge part. The input vector of wavelet neural network of flat part usually is a 25 neighborhood of a certain point, and the output vector is this point itself.

(2) Input the training vector pairs $\left(X^{p}, D^{p}\right)$ and $\left(X^{\prime p}, D^{\prime p}\right)$ formula of wavelet neural network. Network parameters, weight matrix of hidden layer and threshold matrix of output layer can be obtained. Then wavelet neural network model of flat part and edge part can be obtained.

(3) Divide the degraded image $\boldsymbol{g}$ into flat and edge parts by using amoeba operator, then input the flat part and the edge part to neural network model of flat part and edge part respectively which have been trained well. Original image estimation $\hat{\boldsymbol{f}}$ can be obtained based on the combination of original image estimations of the flat and edge parts which have been obtained by the two models.

\section{THE SIMULATION EXPERIMENT OF SEA CLUTTER IMAGE RESTORATION TECHNOLOGY}

Combined the neural networks with the morphology, the image restoration technology which could be used in suppressing the wave clutter has been introduced in the last sections. In the following, some simulations will show the effectiveness of the image restoration technology and wave image data measured by the IPIX radar is used in the simulations. The wave image restoration technology is used to remove the noise in the wave image via the image restoration technology. The effect of the wave restoration technology could be verified in two aspects: the clarity difference and the SNR (signal to the ratio) difference.

\section{A. The image suppression of the sea clutter}

Fig.3 shows a piece of sea wave image from sea clutter image data measured by the IPIX radar. And Fig.4.displays the original image and the sea clutter image recovered by the image restoration technology which is based on the wavelet neural network and the mathematical morphology. Fig.4 shows the comparison of the sea clutter images handled by the algorithm listed in this paper and other different algorithms. the last image is the restoration image by WNN in the fig 5 .

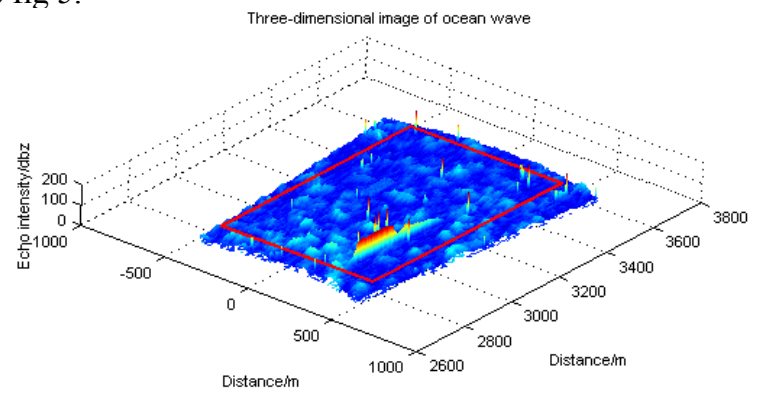

Figure 4. Three-dimensional image of the ocean wave
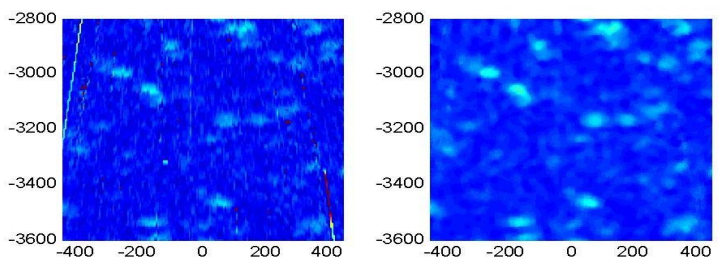

Figure 5. Comparison of the original sea clutter image and the image restored by image restoration technology based on the wavelet neural network and the mathematical morphology
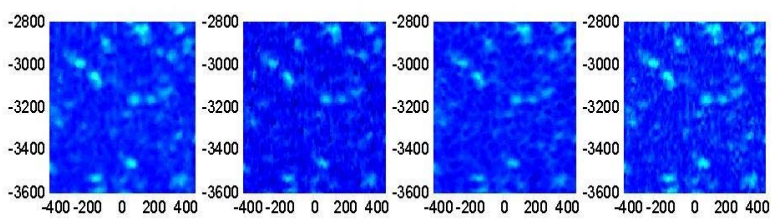

Figure 6. Comparison of sea clutter image obtained by four kinds of image restoration technology

It is clearly observed from Fig. 5 that the effect of the sea clutter image restored by the image restoration technologies based on wavelet neural networks and mathematical morphology is best.

According to the data statistics of the recovered sea images, the amplitude histogram of sea image could be drew out. And the fact is that the amplitude histogram of sea image based on wavelet neural networks and mathematical morphology is closest to the original one. From Fig.7, we know that the image restoration method in this paper is 
effective. And the last image is the restoration image by WNN in the fig 7 and fig 8.

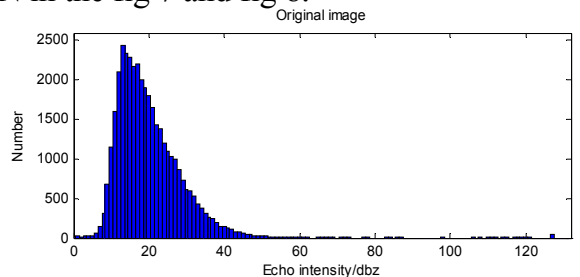

Figure 7. Comparison graph of sea clutter wave image obtained by four kinds of image restoration technology
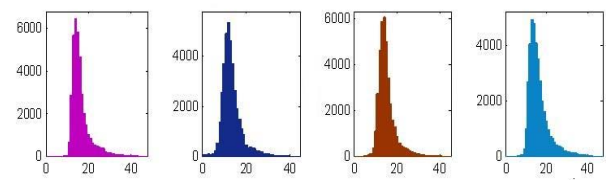

Figure 8. Comparison of the amplitude histogram of sea image obtained by four kinds of image restoration technology

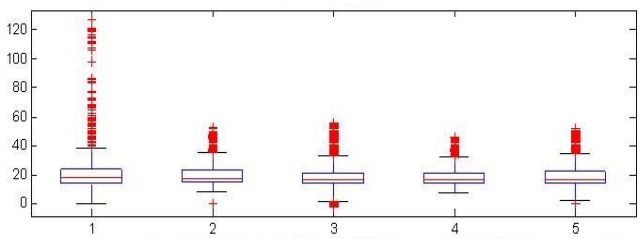

Figure 9. Box graph of sea clutter image obtained by four kinds of image restoration technology

TABLE I. COMPARISON OF THE RESTORATION QUALITY TARGET WITH THE SEA CLUTTER IMAGE

\begin{tabular}{|l|l|l|l|l|}
\hline & $\begin{array}{l}\text { Wavelet } \\
\text { neural } \\
\text { networks }\end{array}$ & $\begin{array}{l}\text { Hopfield } \\
\text { neural } \\
\text { networks }\end{array}$ & $\begin{array}{l}\text { Chaos } \\
\text { neural } \\
\text { networks }\end{array}$ & $\begin{array}{l}\text { Cells } \\
\text { neural } \\
\text { networks }\end{array}$ \\
\hline $\mathrm{NMSE} / \mathrm{dbz}$ & 0.0874 & 0.0952 & 0.1100 & 0.0918 \\
\hline $\mathrm{SNR} / \mathrm{dbz}$ & 10.5835 & 10.2145 & 9.5854 & 10.3708 \\
\hline $\mathrm{PSNR} / \mathrm{dbz}$ & 25.6899 & 25.3209 & 24.6918 & 25.4772 \\
\hline
\end{tabular}

From Table 1, we know that sea clutter restoration image based on wavelet neural networks and mathematical morphology has the minimum normalized mean square error, the highest SNR and the best recovery quality.

\section{CONCLUSIONS}

A new approach is proposed to study the sea clutter image from the image processing point of view in this paper, which circumvents the strong correlation of the sea clutter signal appearing in the conventional sea clutter image restoration techniques. This method combines theories of a lot of subjects such as neural network, mathematical morphology, image restoration, and is of significant innovation and practicability. As for the perspective of image restoration, we improve the conventional neural networkbased image restoration technology. Based on the theory of mathematical morphology, the new approach can effectively retain image edge and details. From the simulation experiment, it can be concluded that the proposed technologies can de-noise and restore the sea clutter image. Comparison of the simulation results shows that the image restoration technologies based on wavelet neural networks and mathematical morphology are relative stable. However, these approaches still leave much room for further improvement, which deserves future investigation.

\section{ACKNOWLEDGEMENTS}

This research was supported by the NNSF of PR China under Grant \#11002037 of Harbin Engineering University and the Research Fund from Special Fund of Basic Scientific Research Operating Expenses of Harbin Engineering University (HEUCF20111118).

\section{REFERENCES}

[1] Yan Shen;Guoqiang Li .The Chaotic Neural Network is used to Predict the Sea Clutter Signal. 2009 International Conference on Artificial Intelligence and Computational Intelligence.[J],2009,PP:2530

[2] YI Xu, WEI Feng-feng; JUN Yan-hao; Hwang,H.K. Adaptive radar clutter suppression. OCEANS, MTS/IEEE Conference and Exhibition [J], 2001.5, PP 762-768.

[3] LIU Jing-yao; MENG Hua-dong; ZHANG Hao; WANG Xi-qin. Radar Sea Clutter Suppression and Target Indication with a Spatial Tracking Filter. Tsinghua Science \& Technology [J], 2010.4, PP:228234.

[4] XIE Nan; Henry Leung; CHAN Hing. A Multiple-Model Prediction Approach for Sea Clutter Modeling. Transaction on Geoscience and Remote Sensing [J], 2003.6, PP :1491-1502.

[5] Helmken, H.H; Vanderhill, M.J. Very low grazing angle radar backscatter from the ocean surface. Record of the IEEE 1990 International Radar Conference [J], 1990, PP: 181-188.

[6] Henry Leung; Neville Dubash; XIE Nan. Detection of Small Objects in Clutter Using a Ga-RBF Neural Network. Transactions on Aerospace and Electronic Systems [J], 2002.1, PP;98-118.

[7] HU Jing; TUNG Wen-wen; GAO Jian-bo. Detection of Low Observable Targets Within Sea Clutter by Structure Function Based Multifractal Analysis. IEEE Transactions on Antennas and Propagation [J], 2006.1, PP: 136-143.

[8] Harm W. Melief; Harm Greidanus; Piet van Genderen; Peter Hoogeboom. Analysis of Sea Spikes in Radar Sea Clutter Data. IEEE Transactions on Geoscience and Remote Sensing [J], 2006. 4, PP: 985-993.

[9] ZHANG Jun; Gilbert G.Walter, et al. Wavelet Neural Networks for Function Learning. IEEE Transaction on Signal Processing [J], 1995, 6,PP: 1485-1497. 